How can we expect the general public to have a rational and informed approach to people with schizophrenia when learned journals accept advertisements that promote a product through negative stereotyping? Perhaps our willingness to allow this to happen is in accord with work in the field which suggests that health professionals may have even more negative attitudes to mental disorder than the general public (Jorm et al, 1999). A public campaign to combat stigma is undoubtedly important, but perhaps we should be prepared to examine our own beliefs about serious mental illness as a prelude to changing attitudes in society at large.

Crisp, A. H., Gelder, M. G., Rix, S., et al (2000) Stigmatisation of people with mental illnesses. British Journal of Psychiatry, 177, 4-7.

Jorm, A. F., Korten, A. E., Jacomb, P. A., et al (1999) Attitudes towards people with a mental disorder: a survey of the Australian public and health professionals. Australian and New Zealand Journal of Psychiatry, 33, 77-83.

D. McKay Department of Psychological Medicine, The University of Sydney, Block 4 Level 5, Royal North Shore Hospital, St Leonards, NSW 2065, Australia

\section{Serum cholesterol and parasuicide}

Garland et al (2000) reignited the various controversies on the role of cholesterol in psychiatric disorders. The methodology used was similar to those in previous studies (Asberg et al, 1976) which did not control for the substances used in parasuicide. This may affect the levels of the chemical or metabolites being researched. Garland et al (2000) did not mention the methods used in those parasuicides and whether they would have affected serum cholesterol.

Engelberg (1992) and Block \& Edwards (1987) held contrasting views on the relationship between cholesterol and serotonin uptake. The work by Heron et al (1980) used to support the hyposerotonergic function caused by low cholesterol appeared flawed. The serotonin site labelled by Heron et al (1980) is not the uptake site (Hawton et al, 1993), and therefore changes in brain serotonin content cannot be explained on the basis of their data. Furthermore, the serotonin stored within brain cells is not accumulated from blood but synthesised in situ from L-tryptophan.

Plasma cholesterol is in a dynamic state, entering the blood complexed with lipoproteins that keep it in solution and leaving the blood as tissues take up cholesterol.
High-density lipoprotein (HDL)-cholesterol that transports circulating cholesterol to the liver for clearance plays a crucial role. Excess HDL can result from excess alcohol (Parkes et al, 1989). This increases the amount of cholesterol transported peripherally, causing low serum cholesterol. Alcohol, drugs and poisons are usually involved in parasuicides (Asberg et al, 1976) and low cholesterol level may therefore be due to ethanol misuse or poisoning. It is unlikely that cholesterol would provide the needed answers to parasuicide. It would only reduce this complex human behaviour to a 'matter to mind' paradigm.

Asberg, M., Traskman, L. \& Thoren, P. (1976) 5 HIAA in the cerebrospinal fluid: a biochemical suicide predictor? Archives of General Psychiatry, 33, II93-1197.

Block, E. R. \& Edwards, D. (1987) Effect of plasma membrane fluidity on serotonin transport by endothelial cells. American Journal of Physiology, 253, 672-678.

Engelberg, H. (1992) Low serum cholesterol and suicide. Lancet, 339, 727-729.

Garland, M., Hickey, D., Corvin, A., et al (2000) Tota serum cholesterol in relation to psychological correlates in parasuicide. British Journal of Psychiatry, 177, 77-83.

Hawton, K., Cowen, P., Owens, D., et al (1993) Low serum cholesterol and suicide. British Journal of Psychiatry, 162, 818-825.

Heron, D. S., Shinitzky, M., Herschkowitz, M., et al (1980) Lipid fluidity markedly modulates the binding of serotonin to mouse brain membranes. Proceedings of the National Academy of Sciences of the USA, 77, 7463-7467.

Parkes, J. G., Hussain, R. A. \& Goldberg, D. M.

(1989) Effect of alcohol on lipoprotein metabolism. I.

High density lipoprotein binding. Clinical Physiology and Biochemistry, 7, 269-277.

O. J. Famoroti Lishman Brain Injury Unit, The

Maudsley Hospital, Denmark Hill, London SE5 8AZ

\section{Transcranial magnetic stimulation: asymmetrical excitability and depression}

Maeda et al (2000) have succeeded in demonstrating the interhemispheric asymmetry of motor cortical excitability in major depression, using transcranial magnetic stimulation (TMS). This is an important finding that raises questions not only about the pathophysiology of major depression, but also about the state or trait nature of the results.

In discussing possible explanations for this functional asymmetry the authors consider the activity of inhibitory interneurons between cortical output cells, as proposed by Wasserman et al (1996), but it is not clear whether this mechanism is thought to act within the hemisphere being stimulated. The role of transcallosal inhibitory mechanisms has been demonstrated in schizophrenia (Davey et al, 1997; Boroojerdi et al, 1999) and is likely to be relevant to understanding asymmetrical motor thresholds in depression. In support of this view, Menkes et al (1999) hypothesised that depression is associated with decreased left hemisphere excitability with respect to the right hemisphere. They successfully showed that inhibitory lowfrequency repetitive TMS applied to the right frontal lobe produced a significant antidepressant effect, in contrast to exciting the left frontal lobe by means of fast-frequency repetitive TMS, the antidepressant effects of which have been known for some years.

Furthermore, Maeda et al report mean motor thresholds in the depression group of $41.13 \%$ for the left hemisphere and $37.63 \%$ for the right hemisphere, and in the healthy group of $48.29 \%$ for the left hemisphere and $52.7 \%$ for the right hemisphere. This gives a mean motor threshold of $39.38 \%$ for the depression group and $50.50 \%$ for the controls, which suggests important differences in both absolute threshold and laterality between the groups. Any changes to either of these parameters in subjects recovered from depression, and possibly in their first-degree relatives, not only promises new insights into the pathophysiology of depression, but also may provide clues about the most elusive object, a biological marker for depression.

Boroojerdi, B., Töpper, R., Foltys, H., et al (1999) Transcallosal inhibition and motor conduction studies in patients with schizophrenia using transcranial magnetic stimulation. British Journal of Psychiatry, 175, 375-379.

Davey, N. J., Puri, B. K., Lewis, H. S., et al (1997) The effects of antipsychotic medication on

electromyographic responses to transcranial magnetic stimulation of the motor cortex in schizophrenia. Journal of Neurology, Neurosurgery and Psychiatry, 63, 468-473.

Maeda, F., Keenan, J. P., Pascual-Leone, A. (2000) Interhemispheric asymmetry of motor cortical excitability in major depression as measured by transcrannial magnetic stimulation. British Journal of Psychiatry, 177, 169-173.

Menkes, D. L., Bodnar, P., Ballesteros, R. A., et (1999) Right frontal lobe slow frequency repetitive transcranial magnetic stimulation ( $\mathrm{SF}$ r-TMS) is an effective treatment for depression: a case-control pilot study of safety and efficacy. Journal of Neurology, Neurosurgery and Psychiatry, 67, I13-115.

Wasserman, E. M., Samii, A., Mercuri, B., et al (1996) Responses to paired transcranial magnetic stimuli in resting, active and activated muscles. Experimental Brain Research, 109, 158-163.

B. J. Moore The University Department of Psychiatry, Royal Liverpool University Hospital, Liverpool L69 3GA 